\title{
Estimation of Longitudinal Unsteady Aerodynamics of a Wing-Tail Combination from Wind Tunnel Data
}

\author{
Patrick C. Murphy* \\ NASA Langley Research Center, Hampton, VA, 23681-2199 \\ Vladislav Klein ${ }^{\dagger}$ \\ The George Washington University, NASA Langley Research Center, Hampton, VA, 23681-2199
}

This paper presents an initial step toward model identification from wind tunnel data for an airliner configuration. Two approaches to modeling a transport configuration are considered and applied to both steady and large-amplitude forced-oscillation wind tunnel data taken over a wide range of angles of attack. Only limited conclusions could be drawn from this initial data set. Although model estimated time histories of normal force and pitching moment agree reasonably well with the corresponding measured values, model damping parameters did not, for some cases, have values consistent with small amplitude oscillatory data. In addition, large parameter standard errors implied poor information content for model structure determination and parameter estimation. Further investigation of the modeling problem for more general aerodynamic models is recommended with close attention to experiment design for obtaining parameters with high accuracy.

\section{Nomenclature}

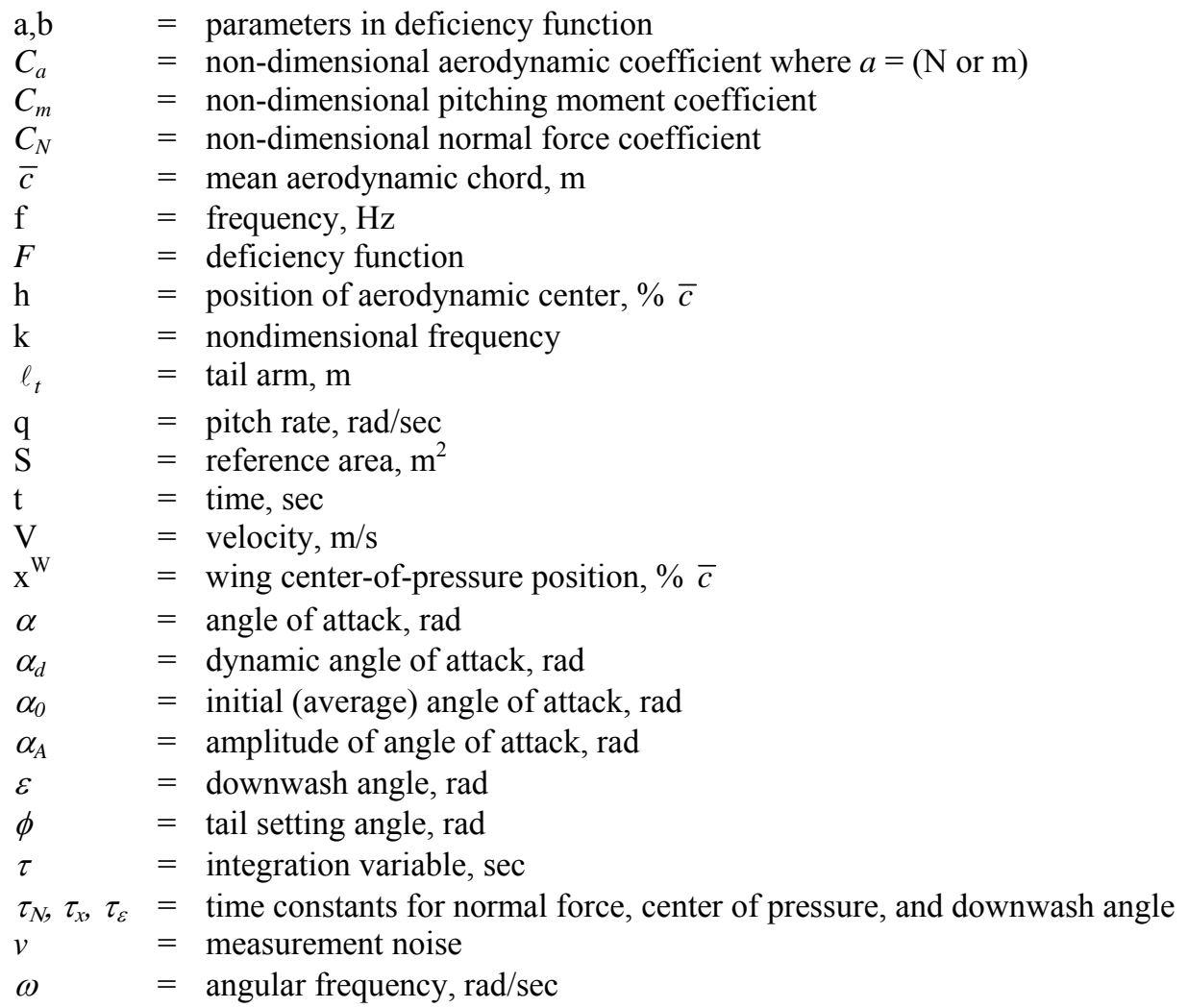

\footnotetext{
* Senior Research Engineer, Dynamic Systems \& Control Branch, Mail Stop 308, Associate Fellow.

${ }^{\dagger}$ Professor Emeritus, Dynamic Systems \& Control Branch, Mail Stop 308, Associate Fellow.
} 


$$
\begin{array}{ll}
\text { subscripts } & \\
A & =\text { amplitude } \\
E & =\text { measurement } \\
\mathrm{t} & =\text { tail } \\
\alpha \mathrm{W} & =\text { wing angle of attack }
\end{array}
$$$$
\text { superscripts }
$$$$
\mathrm{B}, \mathrm{T}, \mathrm{W}=\text { body, tail, wing components }
$$$$
\mathrm{BT}, \mathrm{BW}=\text { body-tail, body-wing components }
$$$$
=\text { estimate }
$$

Aerodynamic Derivatives

$$
\begin{aligned}
& C_{a_{\alpha}}=\frac{\partial C_{a}}{\partial \alpha} \text { where } a=N \text { or } \mathrm{m} \\
& C_{a_{q}}=\frac{\bar{c}}{2 V} \frac{\partial C_{a}}{\partial q} \text { where } a=N \text { or } \mathrm{m}
\end{aligned}
$$

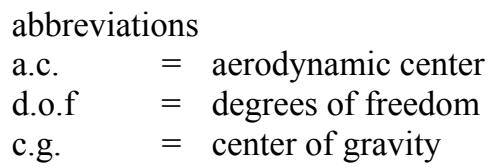

\section{Introduction}

$\mathrm{R}$ ECENTLY there has been increased interest in identification of aircraft aerodynamic models for transport configurations that include unsteady aerodynamic terms. One problem identified under the Aviation Safety Program of NASA is that of developing mathematical models that allow prediction of aircraft response over a large flight envelope including upset conditions. This leads to the problem of developing adequate mathematical models, and methods for their identification, in flight regimes with nonlinear and unsteady aerodynamic behaviors. The majority of models identified to date have been developed for the longitudinal motion of tailless aircraft, (see Refs. 1-3), where the aerodynamic models had relatively simple form. In formulating aerodynamic model equations for a wing-tail combination, however, more complicated model forms can be expected. This problem was first addressed in the early twenties by Cowley and Glauert ${ }^{4}$. They realized that there was a time lag before an aerodynamic disturbance over the wing reaches the tail. They assumed that the downwash associated with a change in lift is equal to the corresponding steady value but the effect at the tail is delayed by the time for the airplane to travel a distance equal to the tail arm. The change of the lift on the tail due to downwash was included in the damping-in-pitch derivative. Now this change is interpreted as a tail contribution to the acceleration derivatives, i.e., the derivative of the lift and pitching moment with respect to rate of change in the angle of attack.

The investigation of the downwash angle was extended by R. T. Jones and Fehlner ${ }^{5}$ by considering both the growth of wing circulation and the delay in the development of lift by the tail. They presented an expression for the downwash indicial function associated with a change of the lift on the wing. Considerable effort to the rigorous explanation of the downwash both qualitatively and analytically was given by Tobak ${ }^{6}$. He developed indicial functions for the lift and pitching moment of a wing-tail combination and presented numerical results for several representative cases in supersonic flight regimes. The theoretical results were then applied to the longitudinal stability analysis of an aircraft.

The linear aerodynamic equations for a planar motion of an aircraft with horizontal tail were developed by Klein ${ }^{7}$. Unsteady effects in these equations were expressed by linear indicial functions for wing and tail aerodynamics, and for the downwash angle. The emphasis was given on model structure which would explain the aerodynamics of wing-tail combination with sufficient accuracy and, at the same time, should be simple enough to provide good conditions for parameter identifiability from experimental data.

A different model of the wing-tail combination was proposed by $\mathrm{Khrabrov}^{8}$ et al, from the Central AeroHydrodynamic Institute (TsAGI). They assume that the aircraft model can be represented by body, wing, and tail components, and that the aerodynamics are, in general, nonlinear and unsteady. The resulting model included algebraic and differential equations with parameters dependent upon the angle of attack. The model was used in the analysis of steady and unsteady wind tunnel data and the identified model was presented in several graphs comparing measured and estimated data. Unfortunately, no numerical values of estimated parameters and their accuracies are given.

The purpose of this paper is to use wind tunnel data from Ref. 8 and aerodynamic model equations based on those of Ref. 7 and 8 for model identification, i.e., model structure determination and parameter estimation. The paper is a product of ongoing research into modeling of an airliner with two lifting surfaces, wing and tail. Results presented in this paper are limited and should be considered preliminary. 


\section{Measured Data}

The measured data were obtained from static and dynamic testing of an airliner in the TsAGI low subsonic wind tunnel. The steady data included the normal force and the pitching moment of four different configurations, i.e., body alone (B), body-tail (BT), body-wing (BW) and the complete model (BWT). These measurements cover the angles of attack from $-10^{\circ}$ up to $30^{\circ}$.

The dynamic data were obtained from a forced oscillatory motion of the same model in the same tunnel using the same test rig. The experiment was executed at different initial values of angle of attack, $\alpha_{0}$, frequencies, and amplitudes, $\alpha_{\mathrm{A}}$. For aircraft model identification, large amplitude $\left(\alpha_{\mathrm{A}}>5^{\circ}\right)$ time histories of $C_{N}$ and $C_{m}$ for three configurations BT, BW, and BWT, at frequencies approximately equal to $0.4 \mathrm{~Hz}, 0.8 \mathrm{~Hz}$, and $1.3 \mathrm{~Hz}$, were used. For large oscillation amplitudes of $15^{\circ}, \alpha_{0}$ was equal to $5^{\circ}$ and $15^{\circ}$ and for oscillation amplitudes of $10^{\circ}, \alpha_{0}$ was equal to $5^{\circ}, 10^{\circ}$, and $20^{\circ}$. Only one cycle of oscillation was given in each time history.

The small amplitude oscillatory data were obtained for $\alpha_{A}=3^{\circ}, \alpha_{0}$ from $-10^{\circ}$ to $30^{\circ}$, frequencies of $0.5 \mathrm{~Hz}, 1.0$ $\mathrm{Hz}$, and $1.5 \mathrm{~Hz}$, and two configurations, BW and BWT. The results are available in the form of the in-phase and outof-phase components $\bar{C}_{N \alpha}, \bar{C}_{N q}, \bar{C}_{m \alpha}$, and $\bar{C}_{m q}$ (see Ref. 1 or 2) and were used for a comparison with identified models. The normal force components are defined as

$$
\begin{gathered}
\bar{C}_{N_{\alpha}}=C_{N_{\alpha}}-k^{2} C_{N \dot{q}} \\
\bar{C}_{N_{q}}=C_{N_{q}}+C_{N \dot{\alpha}}
\end{gathered}
$$

and a similar expression can be written for $\bar{C}_{m \alpha}, \bar{C}_{m q}$. Because of the limited number of frequencies, the data cannot be used for parameter estimation of $C_{N \alpha}, C_{m \alpha}, C_{N q}$, and $C_{m q}$. A more detailed description of the test facility, test conditions, and results are given in Ref. 8.

\section{Analysis of Steady Data}

Modeling the steady data of Ref. 8 took into account the form of measured data. It is assumed that the aerodynamics of the complete aircraft can be formulated as the sum of three contributions due to the body, the tail, and the wing. Then the corresponding models have the form

$$
\begin{gathered}
C_{N}(t)=C_{N}^{B}(\alpha)+C_{N}^{W}(\alpha)+\bar{S}_{t} C_{N}^{T}\left(\alpha_{t}\right) \\
C_{m}(t)=C_{m}^{B}(\alpha)+C_{m_{0}}^{W}+C_{N}^{W}(\alpha) x^{W}(\alpha)-\bar{\ell}_{t} \bar{S}_{t} C_{N}^{T}\left(\alpha_{t}\right)
\end{gathered}
$$

where

$$
\alpha_{t}=\alpha+\phi-\varepsilon(\alpha)
$$

$\bar{S}_{t}=S_{t} / S, \bar{\ell}_{t}=\ell_{t} / \bar{c}$, and $x^{W}$ is the distance between the wing center of pressure and aircraft c.g. as a fraction of mean aerodynamic chord. Eqns. (3) and (4) are taken from Ref. 8 with slightly different notation. 


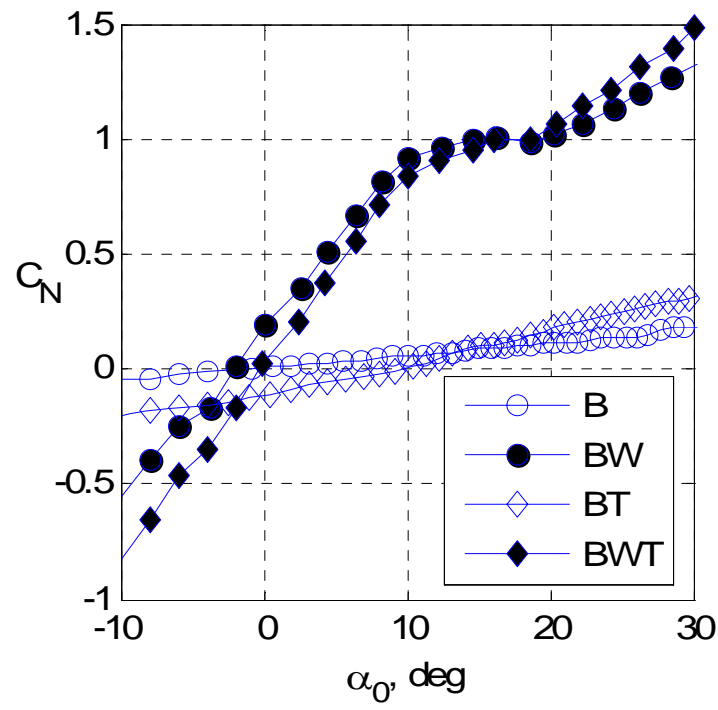

Figure 1. Normal-force coefficient for four configurations (reproduced from Ref. 8).

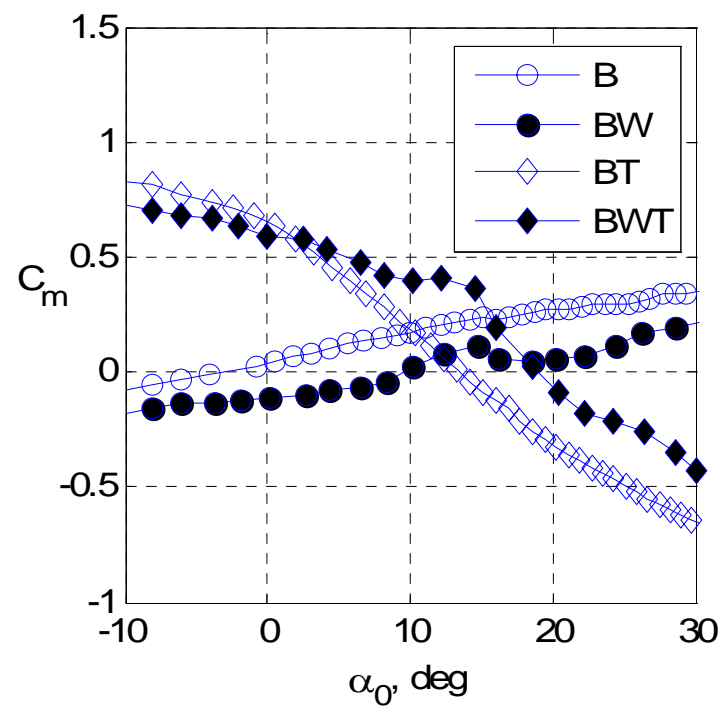

Figure 2. Pitching-moment coefficient for four configurations (reproduced from Ref. 8).

The values of $C_{N}$ and $C_{m}$ for a complete model and its components are reported from Ref. 8 in Fig. 1 and 2 . In these plots B, BW, BT, and BWT indicate the values for the body, body-wing, body-tail, and the complete model. The values of $C_{N}^{W}$ and $C_{N}^{T}$ in Eqs. (3) and (4) were obtained from the relations

$$
\begin{gathered}
C_{N}^{W}=C_{N}^{B W}-C_{N}^{B} \\
C_{N}^{T}=C_{N}^{B T}-C_{N}^{B}
\end{gathered}
$$

Furthermore in Eqns. (3-5), $C_{m_{0}}^{W}$ is equal to the wing pitching moment when $C_{N}^{W}$ is equal to zero. $\phi$ is the tail plane setting and $\varepsilon$ is the downwash angle at the tail. From the geometry of the model $\bar{S}_{t}=0.26, \bar{\ell}_{t}=4.62$ and $\phi=-$ $10^{\circ}$ and from $C_{m}^{W}$ the value of $C_{m_{0}}^{W}=-0.13$. The variable $x^{W}(\alpha)$ can be computed by removing terms associated with the tail in Eq. (4) and using the corresponding BW measurements. The downwash angle follows from solving Eqs. (3-5) for $\alpha_{t}$ at selected $\alpha$. The two variables $x^{W}(\alpha)$ and $\varepsilon(\alpha)$ are plotted in Figs. 3 and 4 and compared with results of Ref. 8. The difference between the two pairs is noticeable for $\alpha<5^{\circ}$. The reason for these differences has not been found.

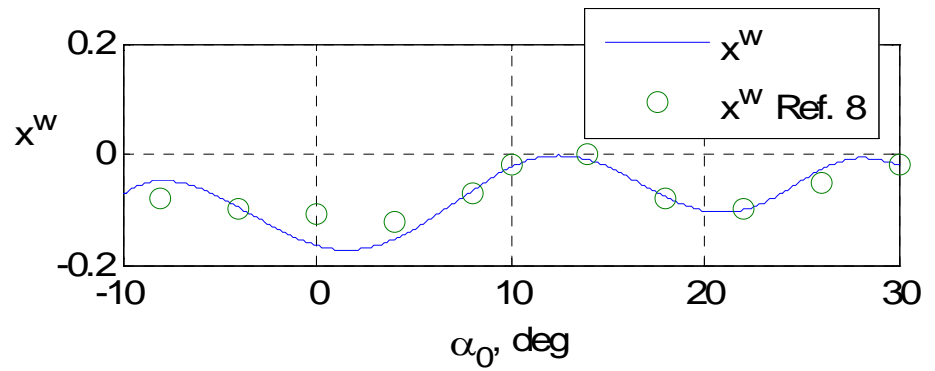

Figure 3. Distance $x^{w}$ estimated from steady wind tunnel data. 


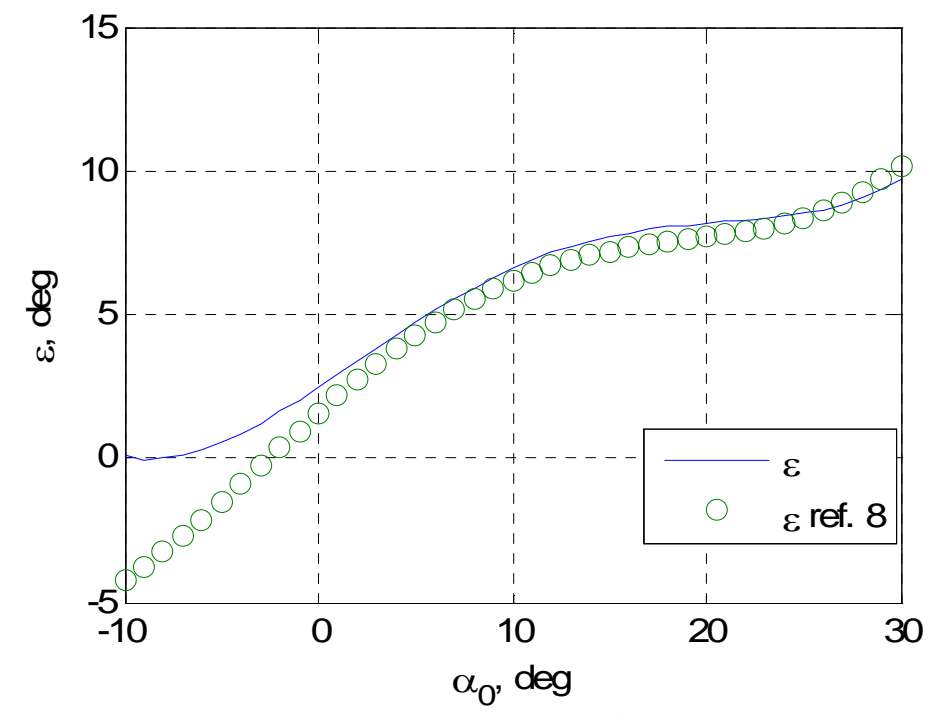

Figure 4. Downwash angle at the tail estimated from steady wind tunnel data.

\section{Model Postulation}

Two model forms will be used in model identification from oscillatory wind tunnel data. The first model, taken from Ref. 8 after minor changes in the notation, will be called the TsAGI model. It has been developed under the following assumptions:

a) For the one d.o.f. harmonic motion in pitch, $C_{N}=C_{N}(\alpha, q)$ and $C_{m}=C_{m}(\alpha, q)$, where $q=\dot{\alpha}$.

b) Aircraft aerodynamics characteristics are obtained by contributions of the body, tail, and the wing.

c) The unsteady effects in the aerodynamic model equations are included in $C_{N}^{W}, x^{W}$, and $\varepsilon$.

Then the model equations can be formed as

$$
\begin{gathered}
C_{N}(t ; \alpha)=C_{N}^{B}(\infty ; \alpha)+C_{N}^{W}(t ; \alpha)+\bar{S}_{t} C_{N}^{T}\left(t ; \alpha_{t}\right)+\frac{\bar{c}}{2 V_{0}} C_{N q}(\infty ; \alpha) \dot{\alpha}(t) \\
C_{m}(t ; \alpha)=C_{m}^{B}(\infty ; \alpha)+C_{m_{0}}^{W}+C_{N}^{W}(t ; \alpha) x^{W}(t ; \alpha)-\bar{\ell}_{t} \bar{S}_{t} C_{N}^{T}\left(t ; \alpha_{t}\right)+\frac{\bar{c}}{2 V} C_{m q}(\infty ; \alpha) \dot{\alpha}(t)
\end{gathered}
$$

where

$$
\begin{gathered}
\alpha_{t}=\alpha+\phi+\alpha_{d}-\varepsilon(t ; \alpha) \\
\alpha_{d}=\frac{\ell_{t} \dot{\alpha}}{V} \\
\tau_{N}(\alpha) \dot{C}_{N S}^{W}+C_{N S}^{W}=C_{N S}^{W}(\infty ; \alpha) \\
\tau_{x}(\alpha) \dot{x}^{W}+x^{W}=x^{W}(\infty ; \alpha) \\
\tau_{\varepsilon}(\alpha) \dot{\varepsilon}+\varepsilon=\varepsilon(\infty ; \alpha)
\end{gathered}
$$

The normal force coefficient due to the wing is partitioned as 


$$
C_{N}^{W}=C_{N A}^{W}+C_{N S}^{W}
$$

where $C_{N A}^{W}$ is the linear part (attached flow) and $C_{N S}^{W}$ is the nonlinear part (separated flow). Symbol $\infty$ indicates a variable evaluated in steady flow. Unknown parameters in the TsAGI model are the time constants, $\tau_{N}, \tau_{x}, \tau_{\varepsilon}$, and two damping terms, $C_{N_{q}}(\infty ; \alpha)$ and $C_{m_{q}}(\infty ; \alpha)$. In general, each of these parameters can be a function of $\alpha$.

The second model, referred to as the LaRC model, was taken from Ref. 7 as

$$
C_{a}(t)=C_{a}(0)+\int_{0}^{t} C_{a_{\alpha}}(t-\tau ; \alpha(\tau)) \dot{\alpha}(\tau) d \tau+\frac{\bar{c}}{2 V} \int_{0}^{t} C_{a_{q}}(t-\tau ; \alpha(\tau)) \dot{q}(\tau) d \tau
$$

where $a=N$ or $m, C_{a}(0)$ is the value of the coefficient during steady conditions, and $C_{a_{\alpha}}(t)$ and $C_{a_{q}}(t)$ are the indicial functions describing the response of $C_{a}(t)$ to a sudden change in $\alpha$ and q.

Sometimes it is more convenient to use the deficiency functions, $F_{a_{\alpha}}(t), F_{a_{q}}(t)$, rather than the indicial functions. Then the two functions are related as

$$
\begin{gathered}
F_{a_{\alpha}}(t)=C_{a_{\alpha}}(\infty ; \alpha)-C_{a_{\alpha}}(t) \\
F_{a_{q}}(t)=C_{a_{q}}(\infty ; \alpha)-C_{a_{q}}(t)
\end{gathered}
$$

where $a=\mathrm{N}$ or $\mathrm{m}$. Substituting Eqs. (17) and (18) into Eq. (16) and replacing $F_{a_{q}}(t)$ by its steady value results in

$$
C_{a}(t)=C_{a}(\infty ; \alpha)+\frac{\bar{c}}{2 V} C_{a_{q}}(\infty ; \alpha) q-\int_{0}^{t} F_{a_{\alpha}}(t-\tau ; \alpha(\tau)) \dot{\alpha}(\tau) d \tau
$$

The indicial function $C_{N_{\alpha}}(t)$, and consequently the deficiency function $F_{N_{\alpha}}(t)$, includes combined responses of the wing and the tail, and interference effects between those two lifting surfaces. It is therefore assumed that the resulting indicial functions are given as a sum of these four components:

\begin{tabular}{|l|l|}
\hline (1) response of $C_{N}^{W}$ to a unit step in $\alpha_{w}$, while $\alpha_{t}=0$. & (2) response of $C_{N}^{T}$ to a unit step in $\alpha_{w}$, while $\alpha_{t}=0$. \\
\hline (3) response of $C_{N}^{W}$ to a unit step in $\alpha_{t}$, while $\alpha_{w}=0$. & (4) response of $C_{N}^{T}$ to a unit step in $\alpha_{t}$, while $\alpha_{w}=0$. \\
\hline
\end{tabular}

For this study, however, it is assumed that the tail does not exhibit an unsteady response and that it does not affect wing response, therefore the third and fourth components (bottom row) are neglected. The first component represents the response of an isolated wing, and the second expresses the lift on the tail due to a change in the downwash induced by the lift of the wing.

As pointed out in Ref. 7, the contribution of the wing and the tail to their indicial functions can be written as

$$
\begin{gathered}
C_{N}(t)=C_{N}^{W}(t)+\bar{S}_{t} C_{N}^{B T}\left(\alpha_{t}\right) \\
C_{m}(t)=C_{m_{\text {a.c. }}}+h C_{N}^{W}(t)-\bar{\ell}_{t} \bar{S}_{t} C_{N}^{B T}(t)
\end{gathered}
$$

Where $C_{m_{\text {a.c. }}}$ is the pitching moment about the aerodynamic center of the wing and $\mathrm{h}$ is the non-dimensional distance between the aerodynamic center and the aircraft center of gravity. For the LaRC model, $C_{N}^{W}$ is located at the aerodynamic center. Combining Eqs. (19-21) yields 


$$
\begin{aligned}
C_{N}(t)= & C_{N}(\infty ; \alpha)+\frac{\bar{c}}{2 V_{0}} C_{N q}(\infty ; \alpha) q(t) \\
& -\int_{0}^{t}\left[F_{N_{\alpha w}}^{W}(t-\tau ; \alpha(\tau))+\bar{S}_{t} F_{N_{\alpha w}}^{B T}(t-\tau ; \alpha(\tau))\right] \dot{\alpha}(\tau) d \tau \\
C_{m}(t)= & C_{m}(\infty ; \alpha)+\frac{\bar{c}}{2 V_{0}} C_{m q}(\infty ; \alpha) q(t) \\
& -\int_{0}^{t}\left[h F_{N_{\alpha w}}^{W}(t-\tau ; \alpha(\tau))-\bar{\ell}_{t} \bar{S}_{t} F_{N_{\alpha w}}^{B T}(t-\tau ; \alpha(\tau))\right] \dot{\alpha}(\tau) d \tau
\end{aligned}
$$

For model identification the deficiency functions in Eqs. (22-23) must be specified. Experience with identification of tailless aircraft indicates that a simple exponential function

$$
F_{N_{\alpha w}}^{W}=a e^{-b t}
$$

can be a good approximation. Formulation of the deficiency function $F_{N_{\alpha w}}^{B T}$, however, is more complicated. A model for the corresponding indicial function, $C_{N_{\alpha w}}^{B T}(t)$, is presented in Ref. 7 for linear aerodynamics combining three effects: (1) change of $C_{N}^{W}$ followed by a change in $\alpha_{w},(2)$ change in $\varepsilon$ at the tail following a change in $C_{N}^{W}$, and (3) a change in $C_{N}^{B T}$ due to a change in $\varepsilon$. The resulting expression for the indicial function is

$$
C_{N_{\alpha w}}^{B T}(t)=-C_{N_{g}}^{B T}(t) \varepsilon(0)-\int_{0}^{t} C_{N_{g}}^{B T}(t-\tau) \dot{\varepsilon}(\tau) d \tau
$$

The function $C_{N_{g}}^{B T}(t)$ represents the lift on the tail during passage of the tail through a step change in downwash that is equivalent to the passage through a sharp-edge gust $\mathrm{t}^{5}$.

The model for the indicial function, $C_{N_{\alpha w}}^{B T}(t)$, is too complicated for model identification. It can be substantially simplified by using the Cowley-Glauert explanation of downwash delay. According to their assumptions the downwash from the wing is delayed from reaching the tail by the time $\Delta t=\ell_{t} / V$. With this assumption, the indicial function can be simplified as

$$
C_{N_{\alpha w}}^{B T}(t)=C_{N_{\alpha w}}^{B T}(\infty)(t-\Delta t)
$$

\section{Model Identification}

In preparation for application of the identification algorithms to measured data, all identification algorithms were validated against simulated data with various signal-noise levels, numbers of cycles of harmonic data, initial parameter error, and number of different frequencies. This analysis indicated that single-cycle harmonic data for each of three frequencies and modest measurement noise levels may contain limited information for identification and prevent convergence of the output error algorithm. In this paper the TsAGI model, given by Eqs. (8-15), was used in model identification. The LaRC model, represented by Eqs. (22-23), was utilized only in its simplified form for the BT configuration. The LaRC model has less pre-defined model structure and therefore requires more information content for identification.

\section{A. BT Model Identification}

The BT model equations were obtained by a simplification of the general equations resulting in

$$
C_{N}(t)=C_{N}^{B}(\alpha)+\bar{S}_{t} C_{N}^{T}\left(\alpha_{t}\right)+C_{N q}^{T}(\alpha) \frac{\dot{\alpha} \bar{c}}{2 V}
$$




$$
C_{m}(t)=C_{m}^{B}(\alpha)-\bar{\ell}_{t} \bar{S}_{t} C_{N}^{T}\left(\alpha_{t}\right)+C_{m q}^{T}(\alpha) \frac{\dot{\alpha} \bar{c}}{2 V}
$$

for the TsAGI model and

$$
\begin{gathered}
C_{N}(t)=\bar{S}_{t} C_{N}^{B T}\left(\alpha_{t}\right)+C_{N q}^{B T}(\alpha) \frac{\dot{\alpha} \bar{c}}{2 V} \\
C_{m}(t)=-\bar{\ell}_{t} \bar{S}_{t} C_{N}^{B T}\left(\alpha_{t}\right)+C_{m q}^{B T}(\alpha) \frac{\dot{\alpha} \bar{c}}{2 V}
\end{gathered}
$$

for the LaRC model. For both sets of equations

$$
\alpha_{t}=\alpha+\phi+\alpha_{d}
$$

Eqns. (27-30) were further simplified by assuming that the damping parameters, $C_{N q}^{B T}$ and $C_{m q}^{B T}$, do not change with $\alpha$. After substituting measured values into the model equations, the least squares method in time domain was used for parameter estimation.

The most consistent results were obtained from Eqs. (29-30) after replacing the term $-\bar{\ell}_{t} \bar{S}_{t} C_{N}^{B T}\left(\alpha_{t}\right)$ by the directly measured pitching moment $C_{m}^{B T}\left(\alpha_{t}\right)$. The resulting parameter estimates are summarized in Table I which

Table I. Estimated damping parameters from large-amplitude oscillatory data, BT configuration.

\begin{tabular}{|c|c|cc|cc|cc|}
\hline$\alpha_{0}, \operatorname{deg}$ & $\alpha_{A}, \operatorname{deg}$ & \multicolumn{2}{|c|}{$C_{N q}^{B T}$} & \multicolumn{2}{|c|}{$C_{m q}^{B T}$} & \multicolumn{2}{|c|}{$-\bar{\ell}_{t} C_{N q}^{B T}$} \\
\hline & & $\hat{\theta}$ & $(\hat{\sigma})$ & $\hat{\theta}$ & $(\hat{\sigma})$ & $\hat{\theta}$ & $(\hat{\sigma})$ \\
\hline 5 & 15 & 3.1 & $(0.82)$ & -15 & $(1.7)$ & -15 & $(3.3)$ \\
\hline 15 & 15 & 2.9 & $(0.99)$ & -15 & $(2.1)$ & -13 & $(4.6)$ \\
\hline 5 & 10 & 3.0 & $(0.98)$ & -12 & $(2.5)$ & -14 & $(4.5)$ \\
\hline 10 & 10 & 2.7 & $(1.40)$ & -12 & $(1.4)$ & -12 & $(6.5)$ \\
\hline 20 & 10 & 1.5 & $(0.49)$ & -17 & $(2.6)$ & -7 & $(2.3)$ \\
\hline
\end{tabular}
includes their mean values, $\hat{\theta}$, and standard errors, $\hat{\sigma}$. The mean values shown were obtained by averaging estimates over 3 different frequencies for given $\alpha_{0}$ and $\alpha_{A}$. The last term in Table I shows values of $C_{m_{q}}^{B T}$ computed from $C_{m_{q}}^{B T}=-\bar{\ell}_{t} C_{N q}^{B T}$. These values are in agreement with direct estimates of $C_{m_{q}}^{B T}$. All parameters were estimated with large standard errors, i.e., low accuracy, suggesting limited information content in the data.

Estimated parameters are also compared with derivatives $\bar{C}_{N q}$ and $\bar{C}_{m q}$ in Figs. 5 and 6. These derivatives were estimated from small amplitude oscillatory data at the frequency of $1.5 \mathrm{~Hz}$. For a comparison of different parameters it is assumed that the difference between $\bar{C}_{N q}$ and $C_{N q}^{B T}$, and $\bar{C}_{m q}$ and $C_{m_{q}}^{B T}$ are caused mainly by parameters $C_{N_{\dot{\alpha}}}$ and $C_{m_{\dot{\alpha}}}$ or their nonsteady counterparts (see Eq. (19) and Ref. 9). The direct comparison of parameters in both figures is not possible but at least some trends in the results can be observed. The parameters in Fig. 5 indicate compatibility between $C_{N q}^{B T}$ and anticipated values of $C_{N q}$ using the relationship $C_{N q}=\bar{C}_{N q}-C_{N_{\dot{\alpha}}}$. A similar argument can be made for the differences between $C_{m_{q}}^{B T}$ and $C_{m q}$ in Fig. 6. 


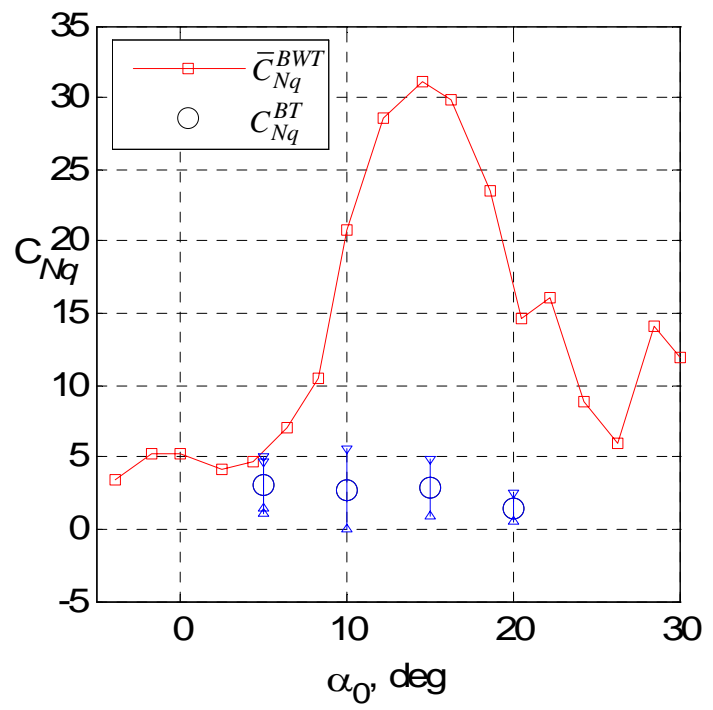

Figure 5. Estimated normal-force damping coefficients due to pitch rate from oscillatory data for BT and BWT configurations.

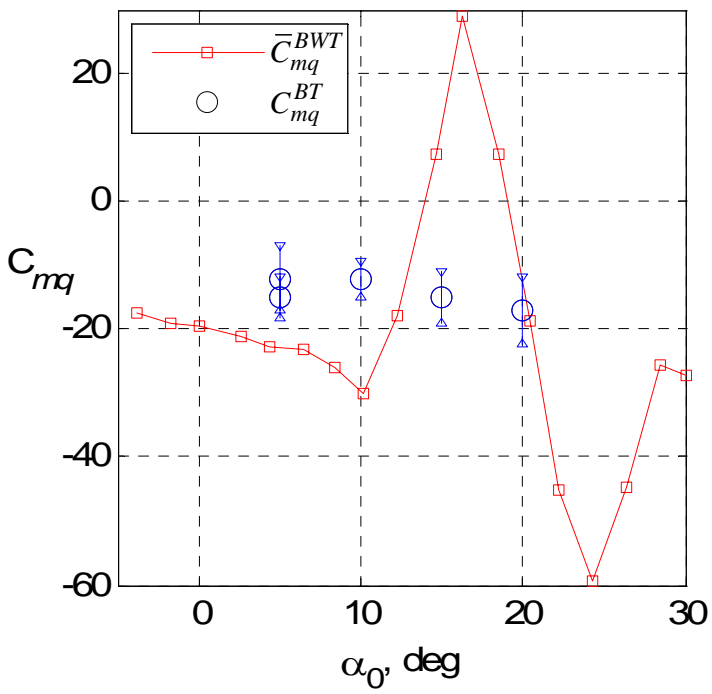

Figure 6. Estimated pitch damping coefficients from oscillatory data for BT and BWT configurations.

\section{B. BW Model Identification}

The BW model equations used in data analysis were taken from the TsAGI model. These equations are

$$
\begin{gathered}
C_{N}(t)=C_{N}^{B}(\infty ; \alpha)+C_{N}^{W}\left(\alpha_{t}\right)+C_{N q}^{B W}(\infty ; \alpha) \frac{\dot{\alpha} \bar{c}}{2 V} \\
C_{m}(t)=C_{m}^{B}(\infty ; \alpha)+C_{m_{0}}^{W}+C_{N}^{W}(\alpha) x^{W}(\alpha)+C_{m q}^{B W}(\infty ; \alpha) \frac{\dot{\alpha} \bar{c}}{2 V}
\end{gathered}
$$

As in the complete model, the normal force coefficient was partitioned as

$$
C_{N}^{W}=C_{N A}^{W}+C_{N S}^{W}
$$

then a time delay due to flow separation can be estimated from

$$
\tau_{N}(\alpha) \dot{C}_{N S}^{W}+C_{N S}^{W}=C_{N S}^{W}(\infty ; \alpha)
$$

Similarly, for identification of the pitching-moment model, the coordinate of the wing center of pressure can be estimated from

$$
\tau_{x}(\alpha) \dot{x}^{W}+x^{W}=x^{W}(\infty ; \alpha)
$$

Now the estimation problem for the $C_{N}$ model parameters can be formulated as follows: given time histories of $C_{N}(t), \alpha(t)$, and $\dot{\alpha}(t)$, and analytical functions $C_{N S}^{W}(\infty ; \alpha), C_{N A}^{W}(\infty ; \alpha)$, and $C_{N}^{B}(\infty ; \alpha)$, estimate unknown constants $\tau_{N}$ and $C_{N q}^{B W}$. To simplify notation, introduce $\eta=C_{N S}^{W}, \theta_{1}=\tau_{N}, \theta_{2}=\frac{\bar{c}}{2 V} C_{N q}^{B W}, u=C_{N S}^{W}(\infty ; \alpha)$, $y=C_{N}(t)-C_{N}^{B}(\infty ; \alpha)-C_{N A}^{W}(\infty ; \alpha)$, then 


$$
\begin{aligned}
& \theta_{1} \dot{\eta}+\eta=u \\
& y=\eta+\theta_{2} \dot{\alpha}
\end{aligned}
$$

A similar formulation can be made for $C_{m}$ where the unknown parameters to be estimated are $\tau_{x}$ and $C_{m q}^{B W}$. The unknown parameters in the state-space models were estimated by an output error method explained in Ref. 10.

Table II. Estimated damping parameters and time constants from large-amplitude oscillatory data, BW configuration.

\begin{tabular}{|c|c|rr|rr|rr|rr|}
\hline$\alpha_{0}, \operatorname{deg}$ & $\alpha_{A}, \operatorname{deg}$ & \multicolumn{2}{|c|}{$\tau_{N}$} & \multicolumn{2}{|c|}{$C_{N q}^{B W}$} & \multicolumn{2}{|c|}{$\tau_{x}$} & \multicolumn{2}{|c|}{$C_{m q}^{B W}$} \\
\hline & & \multicolumn{1}{|c|}{$\hat{\theta}$} & $(\hat{\sigma})$ & \multicolumn{1}{|c|}{$\hat{\theta}$} & $(\hat{\sigma})$ & \multicolumn{1}{|c|}{$\hat{\theta}$} & $(\hat{\sigma})$ & \multicolumn{1}{|c|}{$\hat{\theta}$} & $(\hat{\sigma})$ \\
\hline 5 & 15 & 12.5 & $(1.2)$ & 4.8 & $(2.2)$ & 50.0 & $(3.2)$ & 5.1 & $(.63)$ \\
\hline 15 & 15 & 9.6 & $(1.4)$ & 8.8 & $(3.7)$ & 40.6 & $(2.6)$ & $7.4(.78)$ \\
\hline 5 & 10 & $14.4(1.9)$ & 5.8 & $(2.9)$ & 25.6 & $(1.7)$ & $3.8(.71)$ \\
\hline 10 & 10 & $12.7(1.6)$ & 10.5 & $(4.0)$ & 34.0 & $(1.6)$ & 10.5 & $(.69)$ \\
\hline 20 & 10 & $16.6(1.4)$ & 13.7 & $(4.4)$ & 56.2 & $(.67)$ & 0.2 & $(.67)$ \\
\hline
\end{tabular}

investigated. Large variability of mean values and large standard errors indicates the low accuracy of parameter estimates and limited information content of the measured data.

Damping parameters in Table II were compared in Figs. 7 and 8 with the estimates from small amplitude oscillatory data. Using the same assumptions discussed with Figs. 5 and 6 for comparing the damping estimates with out-of-phase measurements, an agreement between $C_{N q}$ parameters can be argued for $\alpha$ from $-5^{\circ}$ to $5^{\circ}$, and for $\alpha$ near $25^{\circ}$. A large discrepancy in $C_{N q}$ near $\alpha=15^{\circ}$ has not been explained. Small amplitude oscillatory data in Fig. 8 suggests very small negative values would be appropriate for $C_{m q}$ and confirms that the positive values estimated for $C_{m q}$ are incorrect.

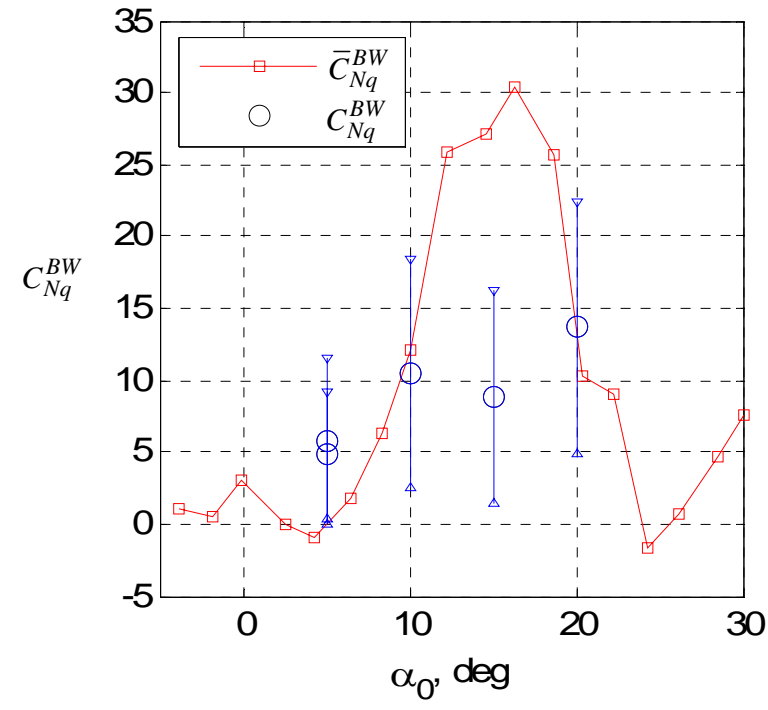

Figure 7. Estimated normal-force damping coefficients due to pitch rate from oscillatory data for BW configuration.

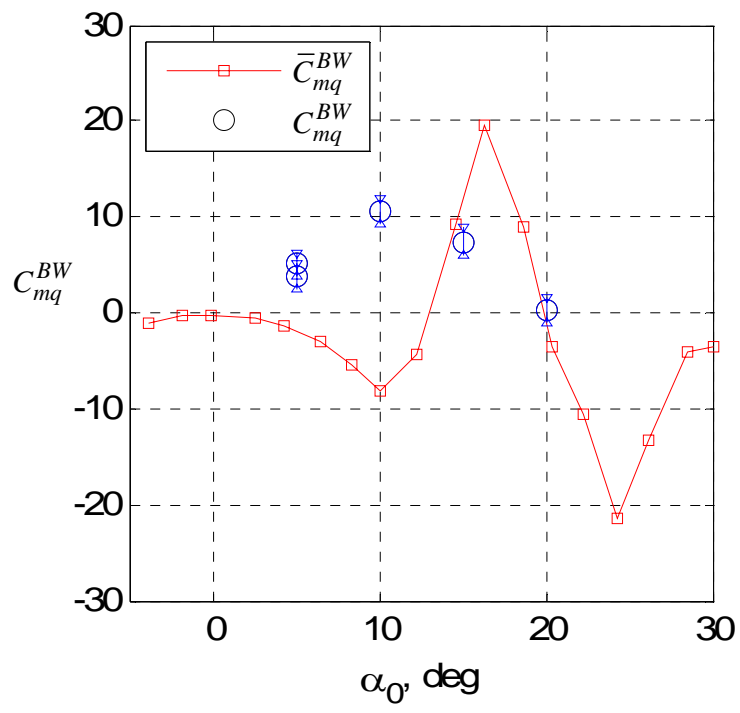

Figure 8. Estimated pitch damping coefficients from oscillatory data for BW configuration.

Measured and computed time histories of $C_{N}$ and $C_{m}$ using parameter values from Table II are plotted in Figs. 9 and 10. Although forced-oscillation experiments were performed separately for each frequency, time history plots 
show all 3 frequency cases stacked into one plot for analysis. In general, there is good agreement between these plots. Some effect of uncorrected model error in $C_{m}$ is visible in part of the data with $\mathrm{f}=0.4 \mathrm{~Hz}$. Figs. 11 and 12 show both coefficients plotted against $\alpha$ with $\mathrm{f}=0.8 \mathrm{~Hz}$. Included in both figures are also steady values of both coefficients. Part of the $C_{N}(\alpha)$ curve for $10^{\circ}<\alpha<20^{\circ}$ with pronounced unsteady effect is captured quite well by identified model despite inaccuracies in both damping parameters. Unexplained differences, however, are apparent in the linear part of $C_{N}(\alpha)$. The correlation between measured and computed $C_{m}(\alpha)$ is poor. Finding an explanation for this requires additional study.

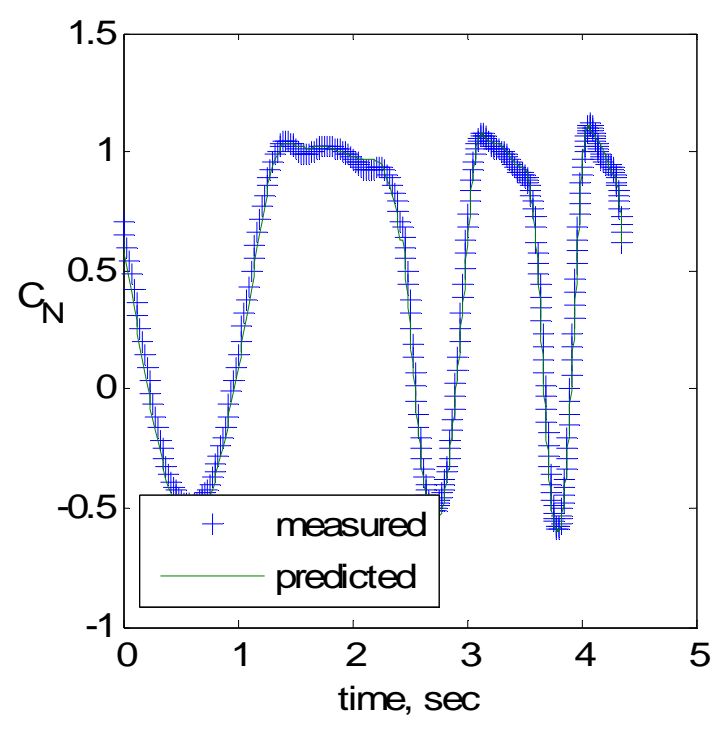

Figure 9. Measured and estimated time histories of normal force for BW configuration, $\alpha_{0}=5^{\circ}$, and $\alpha_{A}=15^{\circ}$.

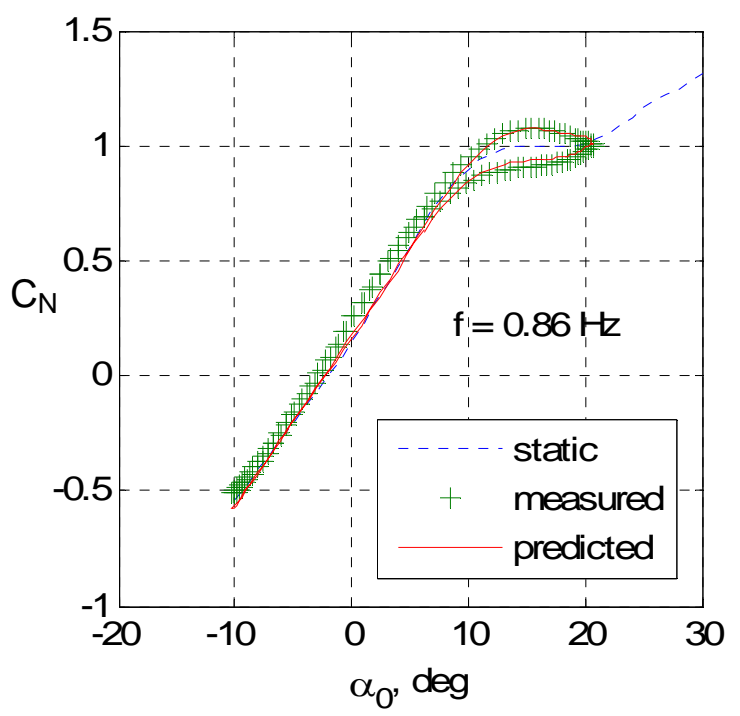

Figure 11. Variation of measured and estimated normal force with $\alpha$ for BW configuration, $\alpha_{0}=5^{\circ}$, and $\alpha_{A}=15^{\circ}$.

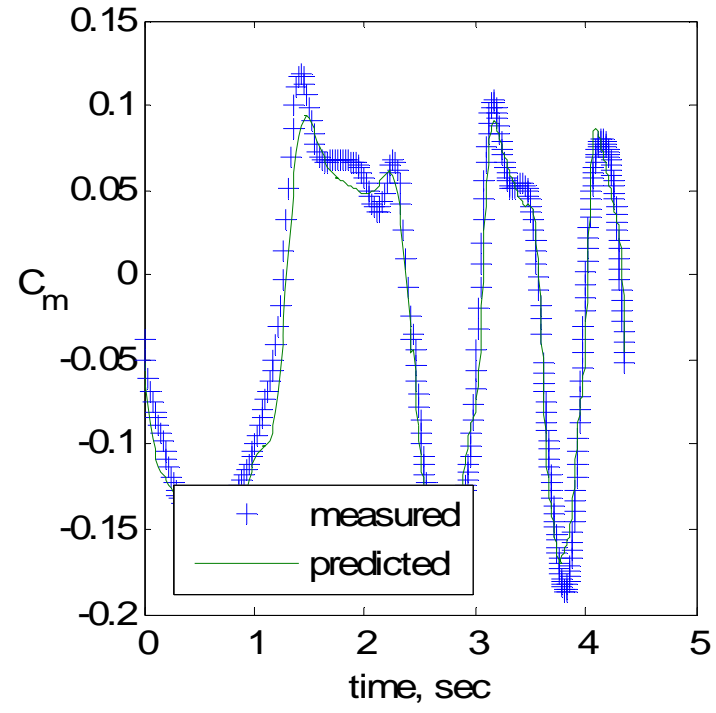

Figure 10. Measured and estimated time histories of pitching moment for BW configuration, $\alpha_{0}=5^{\circ}$, and $\alpha_{A}=15^{\circ}$.

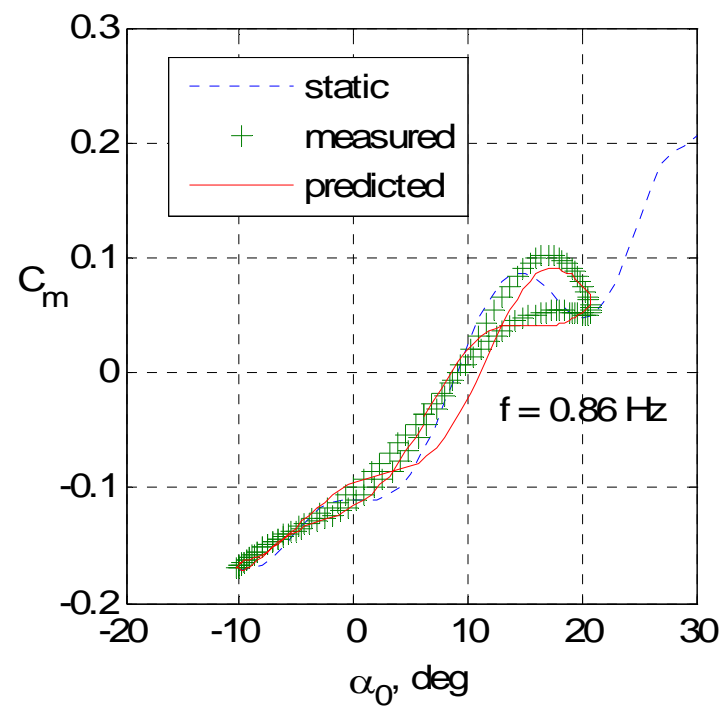

Figure 12. Variation of measured and estimated pitching moment with $\alpha$ for BW configuration, $\alpha_{0}=5^{\circ}$, and $\alpha_{A}=15^{\circ}$. 


\section{BWT Model Identification}

For BWT model identification only the TsAGI model was considered. It is represented by Eqs. (8-15). The unknown parameters in these equations are the two damping derivatives and the time constant, $\tau_{\varepsilon}$, characterizing time dependency of downwash at the tail. The remaining terms in the model equations were obtained from steady data and estimates from BT and BW model identification. As in the previous cases, it was assumed that for each data set the unknown parameters are independent of $\alpha$.

Unknown parameters were estimated using three cycles of oscillatory data combined into one set for a given $\alpha_{0}$ and $\alpha_{A}$. Each cycle in the set was measured at a different oscillation frequency. Estimates of $\tau_{\varepsilon}$ were consistent with an average value of 26 . The parameter estimates for $C_{N q}$ varied from -5 to 4 and for $C_{m q}$ from 14 to 24 . The negative values for $C_{N q}$ and positive values for $C_{m q}$ can't be substantiated. They are also in disagreement with estimates from BT model identification as seen in Table II.

Figures 13 and 14 compare measured and computed coefficients $C_{N}(t)$ and $C_{m}(t)$. The measured data were taken from three cycles at $\alpha_{0}=20^{\circ}$ and $\alpha_{A}=10^{\circ}$. The computed coefficients were based on parameter estimates $\tau_{\varepsilon}=26, C_{N q}=-5$, and $C_{m q}=14$. The agreement between measured and computed coefficients is very good. To investigate the contribution of the damping terms in this case, time histories of $C_{N}$ and $C_{m}$ were recomputed for the same magnitude of damping parameters but with opposite sign, i.e., $C_{N q}=5$ and $C_{N q}=-14$. The resulting values of $C_{N}(t)$ and $C_{m}(t)$ were almost the same as in the preceding computation. These results indicate very low sensitivity of both coefficients, $C_{N}$ and $C_{m}$, to damping parameters for this model and data set. At present, this problem has not been explained. It is expected that further investigation of this problem will continue together with experimental design for obtaining parameters with high accuracy.

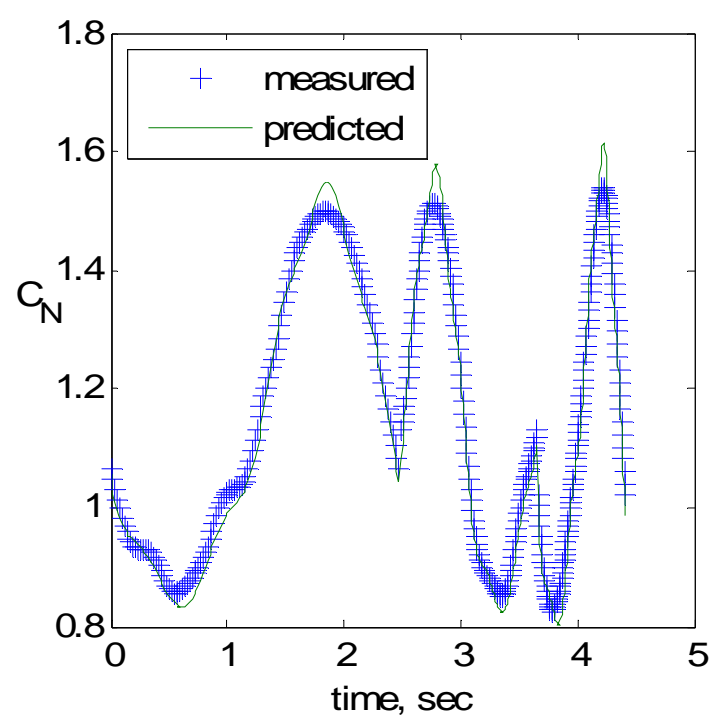

Figure 13. Measured and estimated time histories of normal force for BWT configuration, $\alpha_{0}=20^{\circ}$, and $\alpha_{A}=10^{\circ}$.

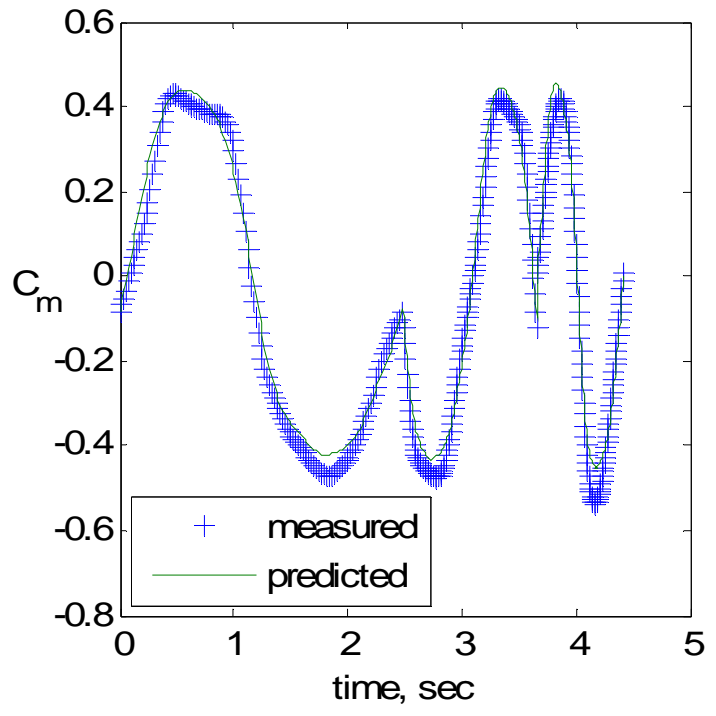

Figure 14. Measured and estimated time histories of pitching moment for BWT configuration, $\alpha_{0}=20^{\circ}$, and $\alpha_{A}=10^{\circ}$.

\section{Concluding Remarks}

This paper presents aircraft model identification from wind tunnel data of an airliner and its components: body, body-tail, body-wing, and the complete model. The measurement data included results from static and oscillatory tests over a wide range of angle of attack. Two mathematical models of an aircraft with one degree-of-freedom in pitch were postulated. These models differ mainly in the formulation of unsteady aerodynamics. 
The measured steady data were fitted by high order polynomials in angle of attack. From these polynomials contributions of the tail and wing alone to normal force and pitching moment were computed followed by determination of downwash angle at the tail and center of pressure position of the wing.

Parameter estimation started with the analysis of the body-tail oscillatory data. After substituting measured data into the model equations for the normal force and pitching moment coefficient, damping parameters in these equations were estimated by a least squares method. The model for the body-wing configuration included four unknowns: two damping parameters and two time constants. These time constants characterize the dynamics defined by differential equations for the unsteady normal force and center of pressure position. After reformulating model equations into a state-space form, the parameters were estimated by an output error method. In the complete model the two time constants from the body-wing analysis were treated as known parameters. The number of unknowns for the complete model analysis was thus reduced to two damping parameters and one time constant for the downwash angle dynamics.

All models with estimated parameters fit the measured data quite well. The estimated time constants for the normal force and downwash were consistent with small deviations from their average values. On the other hand, estimates of the time constant for center of pressure position exhibited large scatter. The value of all three time constants couldn't be verified because there are no references dealing with the same estimation problem.

The estimates of damping parameters of the body-tail and body-wing configurations were compared with the out-of-phase components estimated from small amplitude oscillatory data. The damping parameters agreed with a trend in the out-of-phase results with the exception of the wing contribution to the damping in pitch parameter where those values were positive.

The estimates of both damping parameters in the complete model were mostly unexpected values with positive signs. This is, of course, unacceptable for an aircraft with wing-tail combination operating in the post-stall conditions. It was demonstrated by simulation that both normal force and pitching moment models were insensitive to both damping terms and this was reflected in the identification problem for this test data. Insensitivity of model parameters and large standard errors suggest low information content in the data. It is expected that the ongoing research will explain the problems that occurred in this study and also address the experiment design problem for obtaining parameters with high accuracy in more general aerodynamic models.

\section{Acknowledgment}

The authors are grateful to TsAGI and its scientist, Dr. A. N. Khrabrov for providing the wind tunnel data for this research. Special thanks to Dr. Khrabrov for his assistance in data handling and numerous consultations.

\section{References}

${ }^{1}$ Klein, Vladislav, Murphy, P.C., Curry, T.J., and Brandon, J.M., "Analysis of Wind Tunnel Longitudianl Static and Oscillatory Data of the F-16XL Aircraft," NASA TM-97-206276, 1997.

${ }^{2}$ Klein, Vladislav, and Murphy, P.C., "Estimation of Aircraft Nonlinear Unsteady Parameters From Wind Tunnel Data," NASA TM-98-208969, 1998.

${ }^{3}$ Smith, Mark S., "Analysis of Wind Tunnel Oscillatory Data of the X-31A Aircraft," M.S. Thesis, The George Washington University, Washington D.C., 1998.

${ }^{4}$ Cowley, W. L., and Glauert, H., "The Effect of the Lag of the Downwash on the Longitudinal Stability of an Airplane and on the Rotary Derivative," Mq. R. \& M. 718, 1921.

${ }^{5}$ Jones, Robert T., and Fehlner, Leo F., "Transient Effects of the Wing Wake on the Horizontal Tail,” NACA TN-771, 1940.

${ }^{6}$ Tobak, Murray, "On the Use of Indicial Function Concept in the Analysis of Unsteady Motions of Wings and Wing-Tail Combinations, NACA Rep. 1188, 1954.

${ }^{7}$ Klein, Vladislav, "Modeling of Longitudinal Unsteady Aerodynamics at High Incidence with Account of Wing-Tail Interaction," NASA CR-1999-209547, September, 1999.

${ }^{8}$ Khrabrov, A., Vinogradov, Yu., and Abromov, N., "Mathematical Modeling of Aircraft Unsteady Aerodynamics at High Incidence with Account of Wing-Tail Interaction," AIAA 2004-5278, 2004.

${ }^{9}$ Klein, Vladislav and Norderer, Keith D.: Modeling of Aircraft Unsteady Aerodynamic Characteristics. Part I - Postulated Models, NASA TM 109120, 1994.

${ }^{10}$ Murphy, Patrick C. and Klein, Vladislav: Validation of Methodology for Estimating Aircraft Unsteady Aerodynamic Parameters From Dynamic Wind Tunnel Tests. AIAA 2003-5397, 2003. 